\title{
Epidemiological and Clinical Baseline Characteristics as Predictive Biomarkers of Response to Anti-VEGF Treatment in Patients with Neovascular AMD
}

\author{
Miltiadis K. Tsilimbaris, ${ }^{1}$ Maria I. López-Gálvez, ${ }^{2}$ Roberto Gallego-Pinazo, ${ }^{3}$ \\ Philippe Margaron, ${ }^{4}$ and George N. Lambrou ${ }^{5}$ \\ ${ }^{1}$ Department of Ophthalmology, University of Crete Medical School, Heraklion, 70013 Crete, Greece \\ ${ }^{2}$ Department of Ophthalmology, HCU and IOBA, University of Valladolid, Valladolid, Spain \\ ${ }^{3}$ Unit of Macula, Department of Ophthalmology, University and Polytechnic Hospital La Fe, Valencia, Spain \\ ${ }^{4}$ Novartis Pharma AG, Basel, Switzerland \\ ${ }^{5}$ Vision Institute, National Center of Ophthalmology, Paris, France \\ Correspondence should be addressed to Miltiadis K. Tsilimbaris; tsilimb@med.uoc.gr
}

Received 8 December 2015; Accepted 22 February 2016

Academic Editor: Naoshi Kondo

Copyright ( $\odot 2016$ Miltiadis K. Tsilimbaris et al. This is an open access article distributed under the Creative Commons Attribution License, which permits unrestricted use, distribution, and reproduction in any medium, provided the original work is properly cited.

\begin{abstract}
Purpose. To review the current literature investigating patient response to antivascular endothelial growth factor-A (VEGF) therapy in the treatment of neovascular age-related macular degeneration (nAMD) and to identify baseline characteristics that might predict response. Method. A literature search of the PubMed database was performed, using the keywords: AMD, anti-VEGF, biomarker, optical coherence tomography, treatment outcome, and predictor. The search was limited to articles published from 2006 to date. Exclusion criteria included phase 1 trials, case reports, studies focusing on indications other than nAMD, and oncology. Results. A total of 1467 articles were identified, of which 845 were excluded. Of the 622 remaining references, 47 met all the search criteria and were included in this review. Conclusion. Several baseline characteristics correlated with anti-VEGF treatment response, including best-corrected visual acuity, age, lesion size, and retinal thickness. The majority of factors were associated with disease duration, suggesting that longer disease duration before treatment results in worse treatment outcomes. This highlights the need for early treatment for patients with nAMD to gain optimal treatment outcomes. Many of the identified baseline characteristics are interconnected and cannot be evaluated in isolation; therefore multivariate analyses will be required to determine any specific relationship with treatment response.
\end{abstract}

\section{Introduction}

Age-related macular degeneration (AMD) is the leading cause of blindness in the aging population of industrialized societies [1, 2], responsible for $50 \%$ of cases [3]. Neovascular AMD (nAMD), while representing only $10-20 \%$ of AMD cases, has been reported to be responsible for $80-90 \%$ of severe vision loss and/or legal blindness in this population $[4,5]$.

Vascular endothelial growth factors (VEGF) constitute a family of related molecules with proangiogenic properties (VEGF-A, VEGF-B, VEGF-C, VEGF-D, VEGF-E, and placental growth factor) $[6,7]$. Uncontrolled expression of
VEGF results in growth of new blood vessels that develop abnormalities and fail to mature [6]. This can cause vascular fragility, exudation, and bleeding, as occurs during nAMD [6]. The current standard of care for $\mathrm{nAMD}$ is the intravitreal administration of anti-VEGF-A drugs [8]. Three anti-VEGF therapies that target VEGF-A have been approved for intraocular use in nAMD cases: (1) ranibizumab (Lucentis ${ }^{\circledR}$; Roche Ltd., Basel, Switzerland; Novartis Pharma AG, Basel, Switzerland) [9], (2) aflibercept (Eylea ${ }^{\circledR}$; Regeneron Pharmaceuticals, NY, USA; Bayer Pharma AG, Berlin, Germany) [10], and (3) pegaptanib (Macugen ${ }^{\circledR}$; OSI Pharmaceuticals, NY, USA; Pfizer, NY, USA) [11]. Bevacizumab (Avastin ${ }^{\circledR}$; Roche Ltd., 
Basel, Switzerland) is licensed for use in colorectal cancer but is used off-label to treat nAMD $[8,12]$.

Variation in patient responses to anti-VEGF therapy has been seen in clinical trials. In the MARINA and ANCHOR studies of ranibizumab for the treatment of nAMD, the mean change from baseline in visual acuity (VA) at 24 months for the ranibizumab $0.5 \mathrm{mg}$ group was +6.6 letters and +10.7 letters, respectively $[13,14]$. The majority of patients treated with ranibizumab $0.5 \mathrm{mg}$ achieved improvements or maintained VA at month 24 in both studies, with over $30 \%$ of patients achieving a 15-letter improvement or more from baseline $[13,14]$. However, a small subset of around $10 \%$ of patients lost 15 letters or more from baseline $[13,14]$. This variation in response was also seen in the HARBOR study, where 34.5\% of patients who received monthly ranibizumab $0.5 \mathrm{mg}$ and $33.1 \%$ of patients who received ranibizumab $0.5 \mathrm{mg}$ pro re nata (PRN) gained 15 letters or more at month 24 from baseline [15]. Similar to findings from ANCHOR and MARINA, a small proportion of patients lost 15 letters or more from baseline with both regimens (monthly, 5.8\%; PRN, 9.1\%) [15].

Variation in response to anti-VEGF therapy can also be seen from differences in the frequency of injections required. In the HARBOR study, the median number of injections received by patients in the ranibizumab $0.5 \mathrm{mg}$ PRN treatment arm who completed the study was 14.0 ; however, the range in injection frequency during the 2-year study period was from 3 to 24 injections [15].

Taken together, these data demonstrate that there is variability in patient response to therapy with anti-VEGF agents. Understanding the reasons for this variation could lead to the development of methods to predict individual patient requirements and prevent over- or undertreatment. Attendance at the eye clinic in order to receive intravitreal injections may be inconvenient and expensive for the patient, family, and caregivers; therefore, identifying the optimal injection frequency required without unnecessary clinic visits would be of benefit not only to the patient, but also to the clinic and health system. Measuring factors that could predict patient response to therapy would allow optimization of individualized patient treatment regimens, including frequency and number of injections required, thus reducing the small but real risk of injection-related adverse events, as well as improving disease management and reducing unnecessary monitoring visits.

Aim of the Review. In this paper, we review the current literature investigating patient response to anti-VEGF therapy in the treatment of nAMD and its subtypes and identify clinical baseline characteristics that have been found to predict patient response to anti-VEGF therapy.

\section{Study Design}

A comprehensive search of the literature was conducted using the online biomedical search engine, PubMed. Search terms included age-related macular degeneration; antiVEGF; ranibizumab; bevacizumab; aflibercept; biomarkers, pharmacological, biological markers, angiography; and tomography, optical coherence, treatment outcome, and predictors. Articles and studies were excluded if they met any of the following criteria: articles without full-length versions published in English, reviews, phase 1 clinical trial studies, case reports, and animal studies. Articles focusing on diabetic macular edema, retinal vein occlusion, vitelliform macular dystrophy, retinopathy of prematurity, or oncology also were excluded. The search was performed on June 29, 2015, and all articles meeting the search criteria, from January 2006 onward, were included in this review (Figure 1). The included articles were grouped and reviewed by overall study type (prospective phase 3, prospective nonphase 3, and retrospective studies) with the greatest review weighting given to prospective phase 3 studies (Table 1).

\section{Baseline Factors That Predict Functional and Anatomical Responses to Treatment}

The relationships between a number of baseline characteristics and treatment responses were investigated within the published literature. These broadly fell into the following groups of factors: epidemiological, functional, and anatomical/morphological.

\subsection{Epidemiological Predictive Markers}

3.1.1. Age at Baseline. Retrospective subgroup analysis of the phase 3 MARINA study, which compared ranibizumab and sham treatment in patients with nAMD, used multivariate models to identify age at baseline as a statistically significant predictor of VA outcome at month 24, with increasing age associated with reduced VA gains in both treatment arms [16]. Higher age at baseline was also identified as a statistically significant predictor of worse VA outcome from a similar retrospective subgroup analysis of first-year results from the phase 3 ANCHOR study, which compared ranibizumab and verteporfin photodynamic therapy (vPDT) in patients with nAMD [17]. Patients receiving ranibizumab during MARINA and ANCHOR entered an open-label extension study, HORI$\mathrm{ZON}$, and subsequent long-term follow-up analysis (7-8 years after initial study enrollment) was performed in the SEVENUP study [18]. Comparisons of patient age and final letter score measured during the SEVEN-UP study confirmed that older patients had significantly poorer visual outcomes in this patient population $(p=0.027)$ [18]. A small $(N=31)$ prospective, single-arm, 24-month study using ranibizumab for the treatment of retinal angiomatous proliferation (RAP) also identified a negative correlation between age at baseline and final best-corrected VA (BCVA; $R=-0.357, p=0.049$, Spearman's rho test), although when analyzed using multiple linear regression analysis it narrowly missed significance $(p=0.051)[19]$.

Multivariate analysis of a cohort study within the phase 3 CATT trial of bevacizumab versus ranibizumab in patients with nAMD identified older age at baseline as a predictor of worse VA score at year $1(p=0.0006)$ and less overall VA gain $(p=0.003)$ in both treatment arms [20].

Retrospective analysis of medical records from patients with nAMD treated with ranibizumab supports these phase 


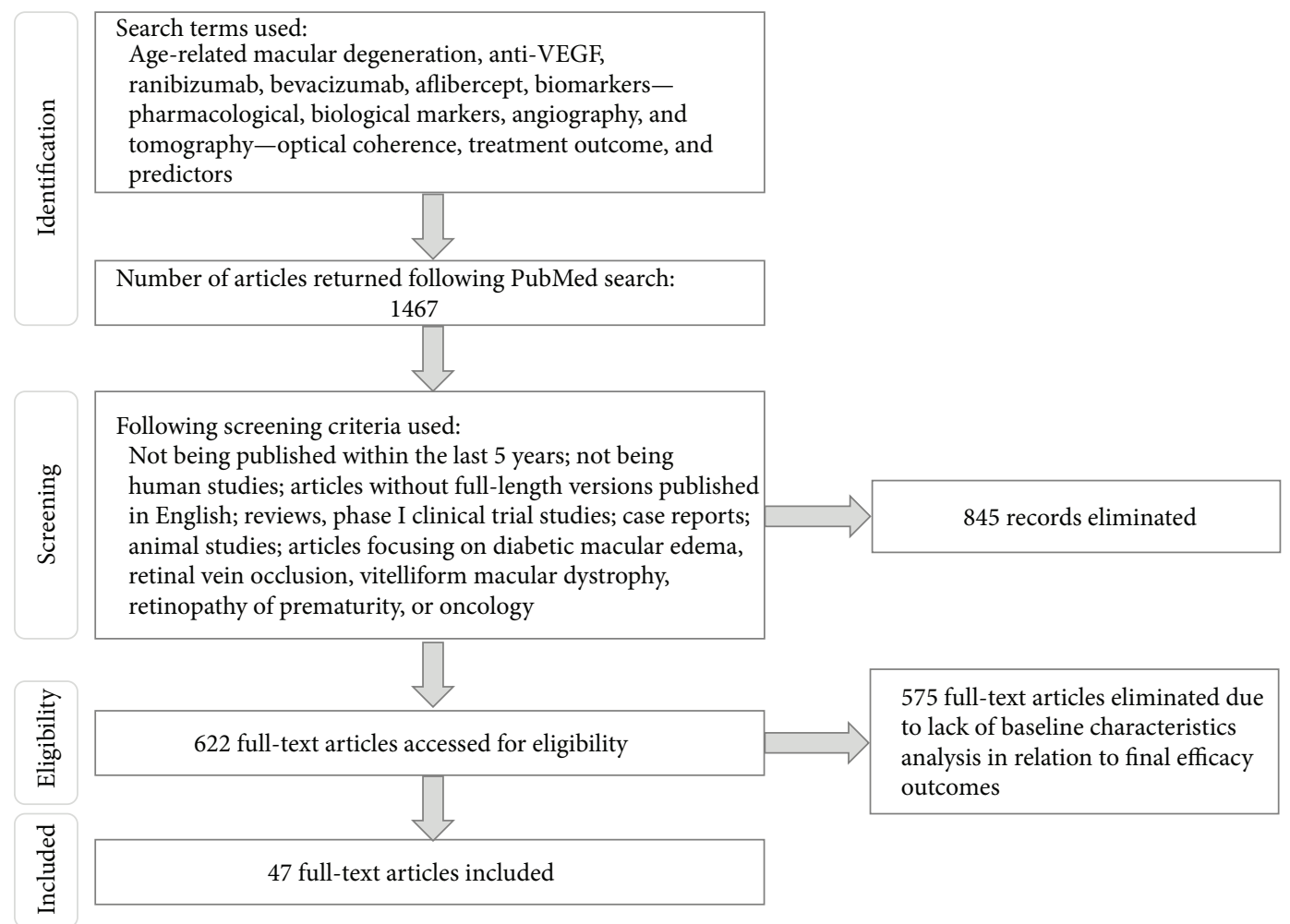

FIGURE 1: Literature search process.

3 data, with a Pearson correlation test identifying increasing age as significantly associated with a worse visual prognosis at month 12 for patients with nAMD ( $p=0.02$ ), but interestingly not those with polypoidal choroidal vasculopathy (PCV; $p>0.22$ ) [21]. However, a separate retrospective interventional cohort study of ranibizumab treatment showed an association between age and response at month 24 in patients with PCV $(p=0.03)$, but not those with nAMD ( $p=0.87)$ using univariate logistic analyses [22]. Age at baseline was also identified as a predictor of VA response at 3 to 12 months in retrospective analyses of patients receiving bevacizumab [23-25] and ranibizumab [26].

3.1.2. Duration of Disease and Previous Treatment. Although not supported by long-term data from large prospective studies, a shorter duration of disease prior to initiating antiVEGF treatment was associated with better VA outcomes at 6 months in two retrospective studies [23, 27].

Similarly, two 6-month retrospective studies identified an association between treatment status at baseline and final outcomes, with treatment-naïve patients achieving a greater reduction in central retinal thickness (CRT) [28] and better VA [24] compared with those who had received prior treatment for nAMD. However, the treatment-naïve status of patients could also be associated with shorter disease duration, but details on disease duration were not recorded and so the actual relevance of treatment status is difficult to determine. Unfortunately there are no data from phase 3 studies to support these findings either way.

\subsection{Functional Predictive Markers}

3.2.1. Best-Corrected Visual Acuity. Multivariate analysis of the MARINA study identified VA at baseline as a significant predictor of VA outcome at month 24, with higher VA at baseline associated with a smaller gain from baseline in VA at month 24 [16]. Similarly, multivariate analysis of baseline VA score for patients in the ANCHOR study showed a high correlation with the change in VA score at month 12 compared with baseline; a higher baseline VA resulted in less gain in VA from baseline at month 12, but a higher overall VA score at month 12 [17]. Indeed, baseline VA was the most influential predictor of VA outcomes at month 12 identified by this analysis [17]. Pooled data from the ranibizumab treatment arms of MARINA, ANCHOR, PIER, and SAILOR were analyzed to identify early ( $\geq 15$-letter gain at month 3 from baseline) and delayed ( $\geq 15$-letter gain at month 12 from baseline) responders [29]. Comparison of baseline characteristics of these two groups using Student's $t$-test showed that early responders had statistically significant lower mean baseline VA compared with delayed responders $(p<0.05)$ in the MARINA and ANCHOR trials, where patients received ranibizumab monthly, but not in the PIER and SAILOR trials, where patients received ranibizumab quarterly and PRN, respectively [29]. No other statistically significant differences in baseline characteristics were identified between the groups [29].

The CATT subanalysis performed by Ying et al. identified worse baseline VA as a statistically significant predictor of 


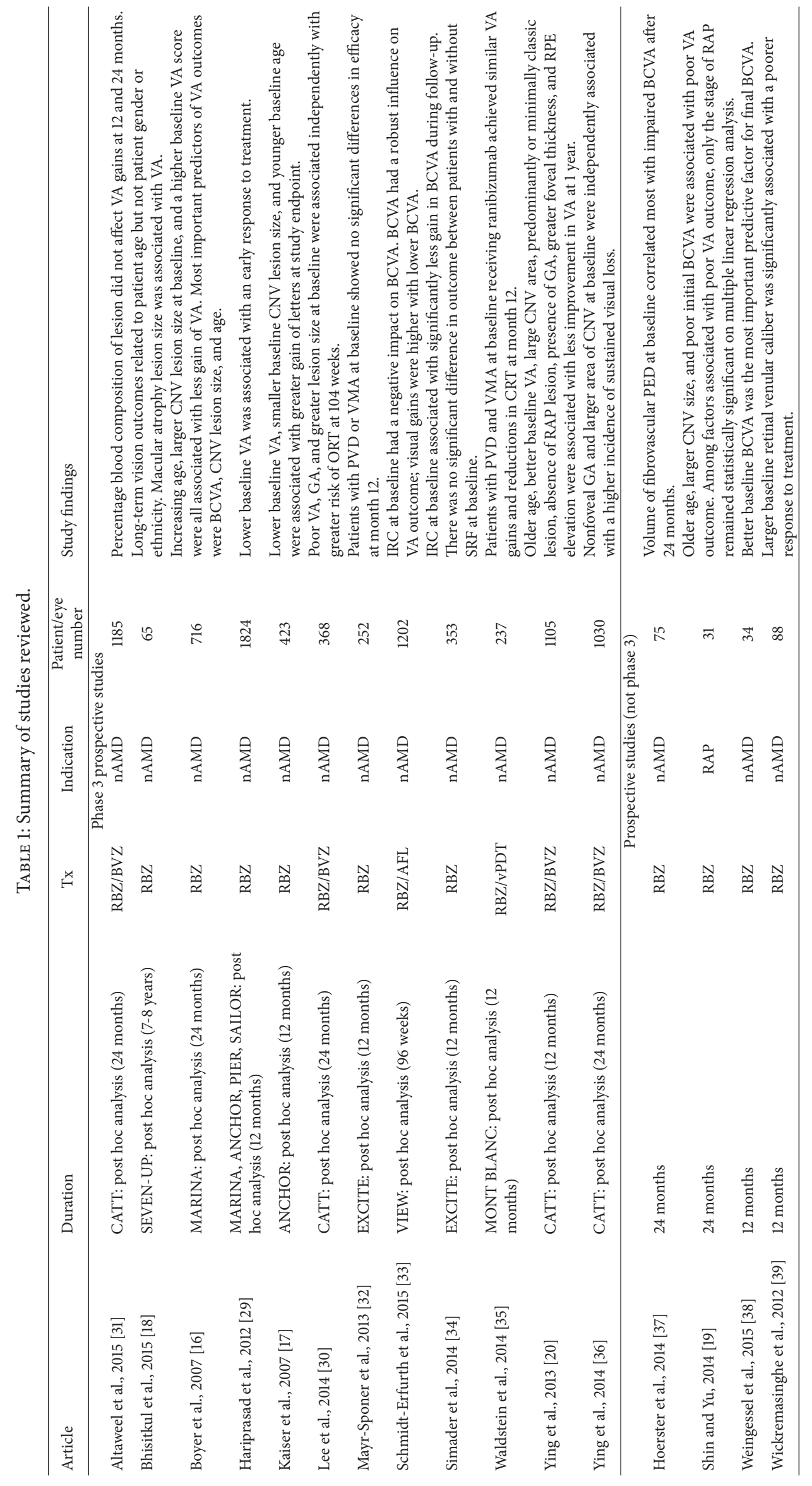




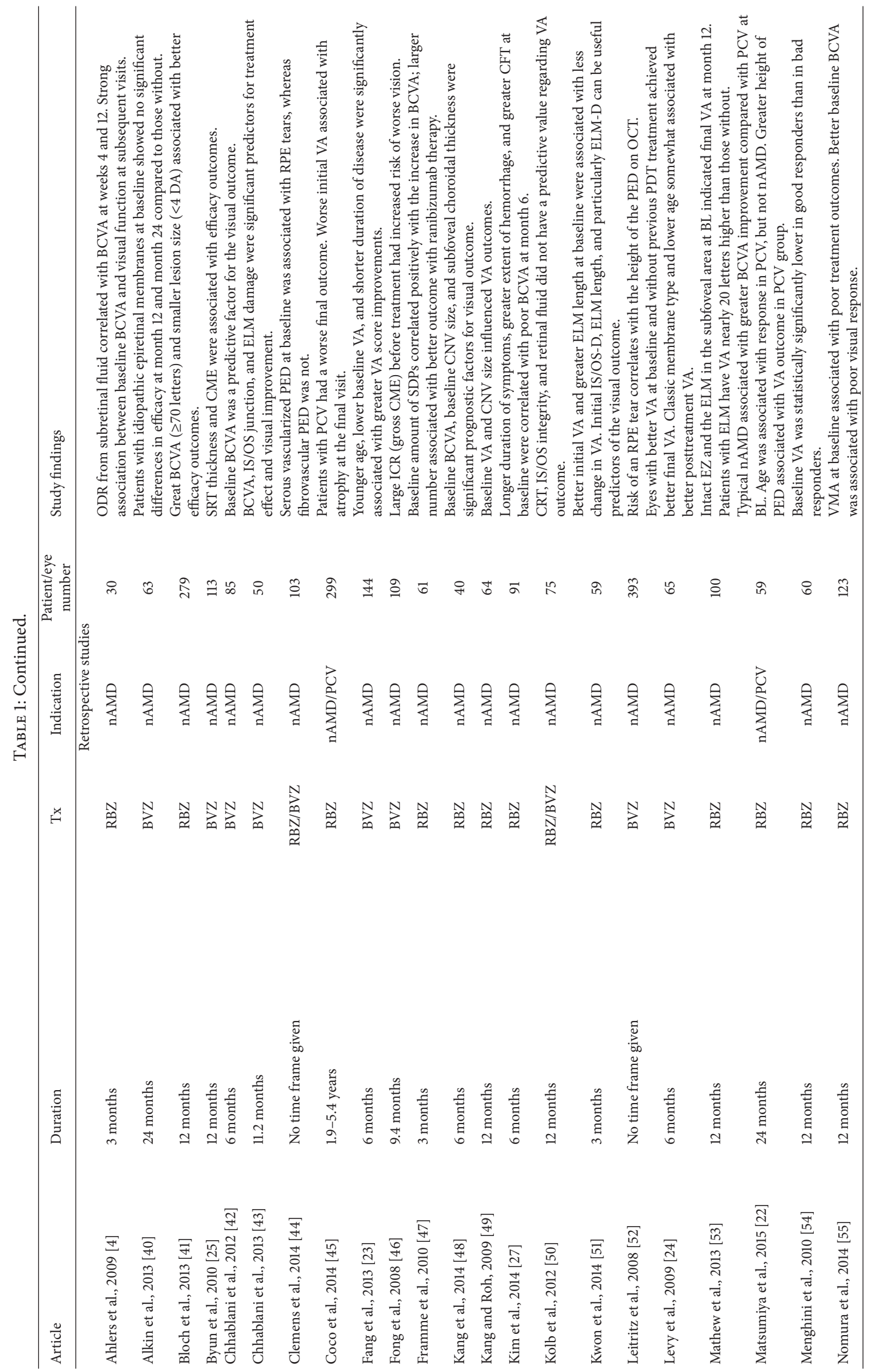




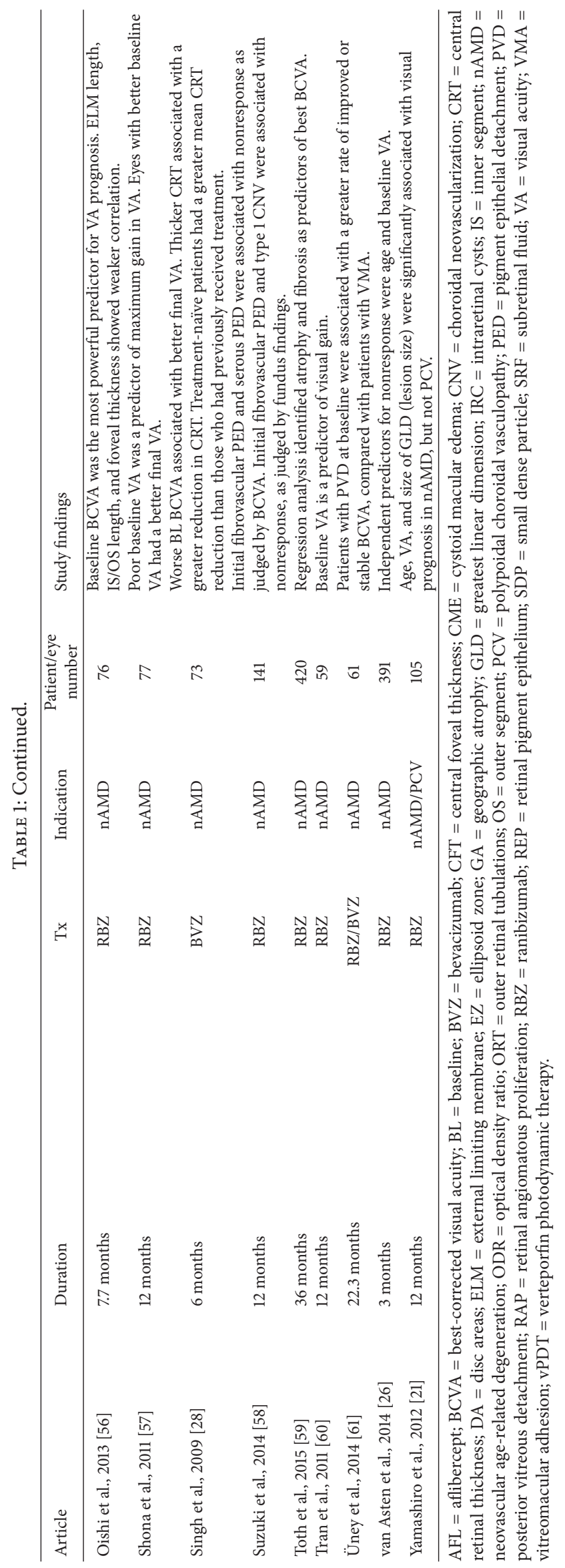


worse VA score at year $1(p<0.0001)$ and a baseline VA of $\geq 20 / 40$ predictive of less VA gain at year $1(p<0.0001)$, irrespective of treatment arm [20]. A separate prospective cohort study of CATT also identified an association between baseline VA and an increased risk of outer retinal tubulations at week 104 ( $p=0.003)$, irrespective of treatment arm [30]. Eyes with outer retinal tubulations at week 104 had worse VA compared with those without [30].

Post hoc analysis of the prospective, phase 3 VIEW study in patients with $\mathrm{nAMD}$ receiving ranibizumab or aflibercept showed a robust influence of baseline BCVA on visual outcomes at week 52 regardless of treatment arm, using a multivariate linear regression model $(p \leq 0.0001)$ [33].

Two small $(N=31$ and $N=34)$ prospective studies of ranibizumab use in patients with nAMD also identified an association between baseline BCVA and final VA outcome at month 12 [38] and month 24 [19].

Retrospective review of medical records supports these phase 3 data and baseline VA was identified as an important predictor of VA outcomes for patients with $\mathrm{nAMD}$ receiving bevacizumab for 6 months [23, 24, 28, 42] and 12 months [43] and for patients receiving ranibizumab for 3 to 6 months $[4,26,48,51,56], 12$ months $[21,41,49,54,55,57,60]$, and 3 to 4 years [45].

\subsection{Anatomical and Morphological Predictive Markers}

3.3.1. Lesion Characteristics. Retrospective analysis of MARINA showed that increased lesion size at baseline was negatively associated with VA outcomes at month 24 $(p<0.0001)$ in response to ranibizumab treatment; for every increase in baseline choroidal neovascularization (CNV) lesion size of 3.6 disc areas, the improvement in VA at the study endpoint was reduced by 5 letters [16]. Subgroup analysis of ANCHOR also identified an association between lesion size and ranibizumab treatment outcome, with smaller lesion size associated with a greater gain in letters at month 12 compared with baseline [17]. Indeed, CNV lesion size was the second most influential predictor of VA outcomes at month 12, after baseline VA, identified by this analysis [17].

$\mathrm{CNV}$ lesion size as a predictor of response to therapy has also been observed with bevacizumab in subanalyses of CATT. Larger CNV lesion size at baseline predicted a lower overall VA score at month $12(p=0.001)$ and reduced gains in VA score at month 12 compared with baseline, irrespective of treatment $\operatorname{arm}(p=0.02)$ [20]. Large lesion size was also associated with an increased risk of outer retinal tubulations at week $104(p=0.01)$ [30]. However, blood content of lesions at baseline did not affect final VA outcomes at years 1 and 2 (lesions composed of $>50 \%$ blood versus $\leq 50 \%$ blood) [31]. A separate subanalysis of CATT assessed patients who developed sustained VA loss at year 2 [36]. Baseline factors independently associated with a higher incidence of VA loss included a larger area of CNV $(p=0.007)$ and presence of nonfoveal geographic atrophy $(p=0.006)$ [36]. These eyes also had more scarring $(p=0.007)$ and hemorrhage $(p=0.03)$, compared with patients without sustained vision loss [36]. Indeed, geographic atrophy at baseline was considered a significant predictor of worse VA outcome at month 12 by multivariate analysis $(p=0.02)$ [20]. Geographic atrophy was also associated with an increased risk of outer retinal tubulations at week $104(p=0.0007)$ [30]. Long-term followup of patients from MARINA and ANCHOR in the SEVENUP study showed that macular atrophy lesion size was the only macular anatomic variable demonstrating a significant association with final VA outcome after 7 to 8 years of treatment, using multivariate linear regression $(p<0.001)$ [18].

Lesion subtype may also predict response to anti-VEGF therapy. A retrospective study of patient medical records after treatment with bevacizumab or ranibizumab for 1 year showed that predominantly or minimally classic lesions were associated with a smaller VA gain than occult lesions $(p=0.0003)[20]$. By contrast, RAP lesions were associated with a greater VA gain $(p=0.03)$ and an increased likelihood of gaining $\geq 3$ lines (OR, 1.9; 95\% CI, 1.2-3.1) after treatment with either bevacizumab or ranibizumab for 1 year [20].

CNV lesion size was also found to be associated with VA outcomes in 3-month [48], 6-month [27], 12-month [41, 49], and 36-month [59] retrospective studies of ranibizumab use in patients with $\mathrm{nAMD}$. Interestingly, the 12-month retrospective review of medical records by Yamashiro et al. reported that larger CNV lesion size at baseline was a prognostic marker for worse VA outcome in response to treatment with ranibizumab in $\mathrm{nAMD}(p=0.0021)$, but not in PCV $(p=0.93)$ [21]. Retrospective review of medical records identified an association between CNV lesion type 1 and nonresponse to ranibizumab according to fundus findings (increase in exudative fundus findings or CRT increase $>100 \mu \mathrm{m}$ after treatment) at month 12 [58].

3.3.2. Vitreomacular Interface. Post hoc analysis of the phase 2 prospective study, MONT BLANC, compared the impact of the vitreomacular interface condition on outcomes of ranibizumab monotherapy versus vPDT plus ranibizumab combination therapy in patients with nAMD [35]. Analysis of variance showed that the change in BCVA from month 3 to month 12 was not significantly different between patients with posterior vitreous detachment (PVD) or vitreomacular adhesion (VMA) in either treatment arm, although combination therapy resulted in a significant loss of potential vision gain in patients with PVD [35]. The vitreomacular interface integrity had no impact on anatomic response to treatment, as measured by change in CRT [35].

Subanalysis of the prospective phase 3 trial, EXCITE, also investigated the effect of the vitreomacular interface on treatment outcomes in patients with $\mathrm{nAMD}$ receiving ranibizumab [32]. Similar to the MONT BLANC analysis, no significant differences were observed in BCVA and CRT outcomes at month 12 between patients with PVD and VMA at baseline [32]. However, ranibizumab monthly dosing conferred benefit over ranibizumab quarterly dosing for patients with VMA, but not PVD [32].

Retrospective review of medical records from patients with nAMD found that VMA at baseline was associated with poor treatment outcomes in response to ranibizumab at month 12 [55] and in response to ranibizumab or bevacizumab after nearly 2 years $(p=0.02)$ [61]. Lastly, the effect of an idiopathic epiretinal membrane on both visual and 
anatomical outcome in response to bevacizumab has been evaluated by a retrospective medical records review and found to have no significant effect at 2 years [40].

3.3.3. Retinal Tissue Thickness. CATT subanalysis showed that greater foveal thickness at baseline predicted a lower overall VA score at month $12(p=0.01)$, irrespective of treatment arm [20]. These findings were supported by retrospective review of medical records, which showed that thicker subretinal tissue, CRT, and foveal thickness at baseline were associated with a reduced visual response after 3 to 12 months of treatment with either bevacizumab or ranibizumab in patients with nAMD $[25,27,28,48,56]$.

3.3.4. Intraretinal Cysts and Cystoid Macular Edema. Subanalysis of the EXCITE study performed by Simader et al. aimed to identify morphologic parameters relevant for visual outcome in patients with nAMD receiving ranibizumab [34]. Correlation analyses demonstrated a significantly lower mean BCVA at month 12 for patients with intraretinal cysts (IRC) at baseline compared with patients without [34].

Post hoc analysis of the VIEW study in patients with $n A M D$ receiving ranibizumab or aflibercept showed that IRC and pigment epithelial detachment (PED) at baseline were predictive of a negative treatment outcome at week 52 regardless of treatment arm, using a multivariate linear regression model ( $p \leq 0.0001$ and $p=0.028$, resp.) [33]. The volume of fibrovascular PED at baseline was also associated with impaired BCVA at month 24 in a separate prospective study of patients with $\mathrm{nAMD}$ receiving ranibizumab $(p=0.011)$ [37].

Retrospective analyses of medical records from patients with $\mathrm{nAMD}$ receiving bevacizumab for 9 to 12 months demonstrated that the presence of cystoid macular edema at baseline was significantly associated with a worse visual outcome $[25,46]$. Retrospective studies also supported the association between fibrovascular PED volume size and visual outcomes for patients receiving ranibizumab [58].

3.3.5. Retinal Vascular Caliber. Retinal vascular caliber can be measured by imaging the fundus and is an indirect indicator of ocular blood flow [39]. Wickremasinghe et al. reported in a prospective study of 88 patients with nAMD receiving ranibizumab that retinal vascular caliber predicted visual outcomes after intravitreal ranibizumab treatment for nAMD [39]. Retinal vascular caliber was separated into central retinal arterial equivalent (CRAE) and venular equivalent (CRVE) to represent the average caliber of arterioles and venules, respectively. Although no correlation was seen between CRAE and visual outcome using multinomial logistic regression analysis, patients experiencing deterioration in VA at 12 months compared with baseline had significantly larger CRVE at baseline (243.10 $\mu \mathrm{m}$; 95\% CI, 227.01-259.19) compared with patients with stable VA $(214.30 \mu \mathrm{m}$; 95\% CI, 205.79-222.81) or improved VA $(215.26 \mu \mathrm{m}$; 95\% CI, 204.69225.84; $p=0.007$ ) [39].

3.3.6. Outer Retinal Structures. The external limiting membrane (ELM) and the photoreceptor ellipsoid zone (EZ), formerly known as inner-segment/outer-segment junction, are markers of the integrity of the photoreceptor layer [43]. Retrospective analysis of medical records from patients with $n A M D$ receiving bevacizumab showed that pretreatment integrity (damage) of both the ELM and EZ was significantly negatively associated with visual response after a mean of 11 months ( $p=0.039$ and $p=0.043$, resp.) using multivariate analysis [43]. Baseline abnormalities of ELM and EZ have both been shown to be negatively associated with visual outcomes at 3 to 12 months in retrospective studies of patients with nAMD receiving ranibizumab [51, 53, 56]. However, a separate retrospective study did not find any association between EZ integrity at baseline and visual outcome at month 12 in response to ranibizumab treatment [50].

Retinal pigment epithelium (RPE) tears can occur spontaneously but can also occur as a serious complication of antiVEGF therapy that may lead to decline or loss of vision. A retrospective evaluation of patients with serous vascular or fibrovascular PED found that RPE tears only developed in patients with serous PED (14.6\%) [44]. The reason for this may be that stretching forces on the weakened RPE result in a tear [52]. In a retrospective chart review of patients with nAMD who received bevacizumab, the risk of an RPE tear increased exponentially with increased height of the PED at baseline [52].

Small dense particles (SDPs) may represent proinflammatory and proangiogenic cells, such as macrophages [47, 62]. Baseline SDPs may predict therapeutic outcomes; for example, in a retrospective study of medical records from patients receiving ranibizumab for $\mathrm{nAMD}$, a significant positive correlation was observed between baseline levels of SDPs and the improvement in BCVA at month 3 (Spearman's correlation coefficient $=0.359 ; p=0.005$ ); however, no correlation was observed between baseline SDPs and CRT [47].

\section{Discussion}

This comprehensive literature review investigated whether any baseline characteristic could be identified that would predict a patient's response to anti-VEGF treatment. Across the different clinical trials, variation in patient response to antiVEGF treatment was observed, in terms of both functional improvement and anatomical response [63-66]. In clinical trials in which an individualized treatment regimen was used, variation was also observed in the mean number of required treatments $[15,66]$. Understanding the reasons for this variation may aid in predicting individual patient requirements and so help manage the patient's expectations in terms of both treatment outcome and burden. Such optimization of the treatment interval and treatment frequency could also prevent any over- or undertreatment and reduce the number of unnecessary monitoring visits. In addition to helping reduce the administrative load on healthcare systems, this would also benefit the patients and their carers, the clinic, and health service providers.

The baseline characteristics investigated encompassed epidemiological, functional, and anatomical categories. Epidemiological factors included the age of the patients, duration of the disease, and any previous treatment received. 
Functional factors included baseline VA, whereas anatomical factors focused on lesion characteristics, the vitreomacular interface, retinal tissue thickness, IRC and edema, ocular blood flow, and structural changes.

Of the several characteristics examined, lower baseline VA was found to correlate with greater VA gain in a large number of studies $[4,16-21,23,24,26,28-30,38,41-43$, $45,48,49,51,54-57,60]$. It has been speculated that this may be mainly due to the larger capacity for improvement in patients with lower VA, whereas patients with higher baseline VA reach a plateau such that VA cannot improve further [49]. A greater VA gain may not be indicative of the absolute end VA; indeed, patients with higher baseline BCVA were found to generally achieve higher VA outcomes compared with patients who had poorer baseline VA, despite the fact that these patients with low baseline VA achieved larger absolute VA gain $[17,20,30]$. This may indicate a need to reevaluate the concept of treatment response/treatment success, where not only the gain in VA but also the end VA is evaluated.

In line with the finding that poorer starting VA is predictive of poorer end VA, was the finding that longer disease duration correlated with worse treatment outcome [23, 27]. It has been postulated that this latter finding may also be due to the mechanism of the nAMD disease progression [67]. As previously mentioned, VEGF increases vascular permeability [68], which facilitates extravasation of plasma proteins and migration of new endothelial cells, resulting in neovascularization and edema development [68]. During disease progression and vessel maturation, pericytes are recruited to the endothelial cells to form a sheath and supply VEGF and other cell survival factors to the proliferating endothelial cells $[67,69]$. New vessels are dependent on VEGF and are therefore sensitive to anti-VEGF treatment, until they acquire a pericyte sheath $[67,69]$. As anti-VEGF therapy influences the unprotected endothelial cells, it causes a decrease in edema and initial improvement in VA [67]. Once protected by pericytes, the neovascular complex is resistant to this VEGF inhibition; this may account for the plateau that is usually observed after initial anti-VEGF treatment, irrespective of the baseline VA [67]. Consequently, if the anti-VEGF therapy is stopped, unprotected endothelial cells may again initiate proliferation in response to VEGF, and the disease progresses [67]. Disease progression can also result in subfoveal fibrosis, particularly in patients with type 2 lesions (classic and predominantly classic CNV) [70]. This fibrous tissue is resilient to anti-VEGF treatment and can cause severe vision loss [70]. Subfoveal fibrosis may already be present at baseline in advanced lesions, which may explain why some lesions do not respond as well as others. In advanced nAMD, the neurosensory retina may be irreversibly damaged and thus fluid resolution following anti-VEGF therapy may only be associated with limited functional gains, regardless of the anatomical improvement.

The recruitment of pericytes is initiated by another growth factor, platelet-derived growth factor-BB (PDGF$\mathrm{BB})$. Based on the mechanism outlined above, inhibition of PDGF-BB would result in the stripping of pericytes from vessels, rendering them sensitive to anti-VEGF inhibition [67]. Furthermore, PDGF-BB is also involved in fibrogenesis, and so its inhibition is expected to result in the reduction of fibrosis formation [71]. The efficacy and safety of an antiPDGF-BB agent (E10030) in combination with ranibizumab have been tested in a large phase $2 \mathrm{~b}$ clinical trial. Indeed, in comparison with ranibizumab monotherapy, dual inhibition resulted in a $62 \%$ additional benefit from baseline [72]. Further phase 3 trials are currently in progress [67].

Further to poorer BCVA and longer disease duration, several other characteristics have also been identified as being associated with poorer response. These include older age of the patient, any previous treatment, larger CNV lesion, and larger retinal tissue thickness (Table 1). It should also be noted that the presence of PCV at baseline had a negative effect on treatment outcomes in some studies [21, 22, 45], but not others [58]. Rather than indicating a specific correlation, this may be due to inherent intercorrelation of all these characteristics with longer disease duration and baseline VA. These findings highlight the absolute need for early treatment of patients with nAMD. Because longer disease duration, as well as several characteristics associated with longer duration of the disease (relatively poor baseline BCVA, older age, previous treatment, lesion size, and retinal tissue thickness), largely correlated with a relatively poorer response, earlier treatment initiation may facilitate a better treatment response. In addition, as previously mentioned, longer disease duration and so more advanced disease may also correlate with more extensive tissue and structural damage, resulting in irreversible VA loss.

4.1. Study Limitations. This review was exploratory in nature, so although limited clinical conclusions can be drawn from evaluation of these studies, it does generate hypotheses that may be confirmed by larger prospective trials. Treatment parameters (e.g., regimen, retreatment criteria) and lesion evaluation techniques differed across the different studies; standardization will be required to determine any specific relationships. Because baseline characteristics are interconnected, further larger scale studies and multivariate analyses will be required to definitively confirm these. Further complexity is afforded by the lack of a uniform definition for a "treatment response." Limited evidence exists to definitively link anatomical and functional responses, the association between a decrease in CRT and improvement in VA $[73,74]$. Although it is generally believed that persistent residual fluid in the retina might have long-term implications for visual outcomes and may lead to irreversible retinal damage $[75,76]$, it is currently unclear whether a complete absence of fluid ("dry retina") should be considered a therapeutic goal. It may be that, similar to VA, rather than the absolute change in retinal thickness, its relation to end retinal thickness may need to be considered in the future. As previously mentioned, the change in VA may not be indicative of treatment response and end VA should be taken into account as well, when evaluating treatment success.

\section{Conclusions}

Neovascular AMD is a multifactorial disease and it is unlikely that a single characteristic will be able to define treatment 
response/outcome. It may be that a tool with multiple parameters could be developed to guide and optimize the treatment of nAMD and help guide patient expectations. This paper reviewed the current literature to investigate whether a patient's response to anti-VEGF therapy could be predicted based on any baseline characteristic. Although several different parameters were identified that correlated with poorer prognosis, the majority of factors were associated with disease duration (i.e., longer disease duration results in worse treatment outcomes), highlighting the clinical importance of early treatment in the natural history of nAMD. As many of the identified baseline characteristics are interconnected and cannot be evaluated in isolation, thorough multivariate analyses will be required to determine any specific relationship with treatment response.

\section{Additional Points}

Baseline characteristics may present a useful tool in predicting patient response to anti-VEGF treatment, helping both patients and clinicians plan appropriately. Markers suggest longer disease duration is associated with worse therapy outcomes, highlighting the importance of early treatment. Extensive multivariate analyses are required to determine specific relationships with treatment response.

\section{Disclosure}

The paper was developed in accordance with the good publications practice guidelines (GPP3).

\section{Competing Interests}

Philippe Margaron is employed by Novartis Pharma AG. George N. Lambrou was an employee of Novartis Pharma AG at the time of paper preparation. Miltiadis K. Tsilimbaris has received financial support from Alcon and Coloplast Mavrogenis, has acted as a consultant for Novartis, and received travel support from Bayer, Novartis, Allergan, Alcon, and Thea. Maria I. López-Gálvez has received financial support from Bayer, Novartis, Thea, and Roche, has acted as a consultant for Novartis and Bayer, and has received honoraria for speaking events from Novartis, Lily, and Boehringer. Roberto Gallego-Pinazo has received financial support from Alcon, Angelini, Bayer, Novartis, Thea, and Roche, has acted as a consultant for Novartis, and has received honoraria for speaking events from Heidelberg Engineering, Bausch and Lomb, and Novartis.

\section{Acknowledgments}

Editorial support was provided by Fishawack Communications Ltd., Oxford, UK; this service was funded by Novartis Pharma AG, Basel, Switzerland.

\section{References}

[1] N. Congdon, B. O'Colmain, C. C. Klaver et al., "Causes and prevalence of visual impairment among adults in the United
States," Archives of Ophthalmology, vol. 122, no. 4, pp. 477-485, 2004.

[2] D. Pascolini, S. P. Mariotti, G. P. Pokharel et al., "2002 Global update of available data on visual impairment: a compilation of population-based prevalence studies," Ophthalmic Epidemiology, vol. 11, no. 2, pp. 67-115, 2004.

[3] S. Resnikoff, D. Pascolini, D. Etya'ale et al., "Global data on visual impairment in the year 2002," Bulletin of the World Health Organization, vol. 82, no. 11, pp. 844-851, 2004.

[4] C. Ahlers, I. Golbaz, E. Einwallner et al., "Identification of optical density ratios in subretinal fluid as a clinically relevant biomarker in exudative macular disease," Investigative Ophthalmology \& Visual Science, vol. 50, no. 7, pp. 3417-3424, 2009.

[5] N. M. Bressler, S. B. Bressler, and S. L. Fine, "Age-related macular degeneration," Survey of Ophthalmology, vol. 32, no. 6, pp. 375413, 1988

[6] N. Ferrara, "Vascular endothelial growth factor and age-related macular degeneration: from basic science to therapy," Nature Medicine, vol. 16, no. 10, pp. 1107-1111, 2010.

[7] M. W. Stewart, "Clinical and differential utility of VEGF inhibitors in wet age-related macular degeneration: focus on aflibercept," Clinical Ophthalmology, vol. 6, no. 1, pp. 1175-1186, 2012.

[8] U. Schmidt-Erfurth, V. Chong, A. Loewenstein et al., "Guidelines for the management of neovascular age-related macular degeneration by the European Society of Retina Specialists (EURETINA)," The British Journal of Ophthalmology, vol. 98, no. 9, pp. 1144-1167, 2014.

[9] Lucentis Summary of Product Characteristics, Novartis Pharma AG, Basel, Switzerland, 2014.

[10] Eylea, Summary of Product Characteristics, Bayer Pharma AG, Berlin, Germany, 2015.

[11] Macugen Prescribing Information, OSI Pharmaceuticals, New York, NY, USA, 2011.

[12] Avastin Summary of Product Characteristics, Roche, Basel, Switzerland, 2013.

[13] P. J. Rosenfeld, D. M. Brown, J. S. Heier et al., "Ranibizumab for neovascular age-related macular degeneration," The New England Journal of Medicine, vol. 355, no. 14, pp. 1419-1431, 2006.

[14] D. M. Brown, M. Michels, P. K. Kaiser, J. S. Heier, J. P. Sy, and T. Ianchulev, "Ranibizumab versus verteporfin photodynamic therapy for neovascular age-related macular degeneration: twoyear results of the ANCHOR study," Ophthalmology, vol. 116, no. 1, pp. 57-65.e5, 2009.

[15] A. C. Ho, B. G. Busbee, C. D. Regillo et al., "Twenty-four-month efficacy and safety of $0.5 \mathrm{mg}$ or $2.0 \mathrm{mg}$ ranibizumab in patients with subfoveal neovascular age-related macular degeneration," Ophthalmology, vol. 121, no. 11, pp. 2181-2192, 2014.

[16] D. S. Boyer, A. N. Antoszyk, C. C. Awh, R. B. Bhisitkul, H. Shapiro, and N. R. Acharya, "Subgroup analysis of the MARINA study of ranibizumab in neovascular age-related macular degeneration," Ophthalmology, vol. 114, no. 2, pp. 246-252, 2007.

[17] P. K. Kaiser, D. M. Brown, K. Zhang et al., "Ranibizumab for predominantly classic neovascular age-related macular degeneration: subgroup analysis of first-year ANCHOR results," American Journal of Ophthalmology, vol. 144, no. 6, pp. 850-857, 2007.

[18] R. B. Bhisitkul, T. S. Mendes, S. Rofagha et al., "Macular atrophy progression and 7-year vision outcomes in subjects from the ANCHOR, MARINA, and HORIZON studies: the SEVEN-UP 
study," American Journal of Ophthalmology, vol. 159, no. 5, pp. 915-924.e2, 2015.

[19] J. Y. Shin and H. G. Yu, "Optical coherence tomography-based ranibizumab monotherapy for retinal angiomatous proliferation in Korean patients," Retina, vol. 34, no. 12, pp. 2359-2366, 2014.

[20] G.-S. Ying, J. Huang, M. G. Maguire et al., "Baseline predictors for one-year visual outcomes with ranibizumab or bevacizumab for neovascular age-related macular degeneration," Ophthalmology, vol. 120, no. 1, pp. 122-129, 2013.

[21] K. Yamashiro, K. Tomita, A. Tsujikawa et al., "Factors associated with the response of age-related macular degeneration to intravitreal ranibizumab treatment," American Journal of Ophthalmology, vol. 154, no. 1, pp. 125-136, 2012.

[22] W. Matsumiya, S. Honda, K. Otsuka et al., "Comparison of the effectiveness and prognostic factors of intravitreal ranibizumab between typical neovascular age-related macular degeneration and polypoidal choroidal vasculopathy over 24 months of follow-up," Ophthalmologica, vol. 234, no. 1, pp. 33-39, 2015.

[23] K. Fang, J. Tian, X. Qing et al., "Predictors of visual response to intravitreal bevacizumab for treatment of neovascular agerelated macular degeneration," Journal of Ophthalmology, vol. 2013, Article ID 676049, 9 pages, 2013.

[24] J. Levy, M. Shneck, S. Rosen et al., "Intravitreal bevacizumab (avastin) for subfoveal neovascular age-related macular degeneration," International Ophthalmology, vol. 29, no. 5, pp. 349$357,2009$.

[25] Y. J. Byun, S. J. Lee, and H. J. Koh, "Predictors of response after intravitreal bevacizumab injection for neovascular age-related macular degeneration," Japanese Journal of Ophthalmology, vol. 54, no. 6, pp. 571-577, 2010.

[26] F. van Asten, M. M. Rovers, Y. T. E. Lechanteur et al., "Predicting non-response to ranibizumab in patients with neovascular agerelated macular degeneration," Ophthalmic Epidemiology, vol. 21, no. 6, pp. 347-355, 2014.

[27] J. H. Kim, Y. S. Chang, J. W. Kim, C. G. Kim, S. J. Yoo, and H. J. Cho, "Intravitreal anti-vascular endothelial growth factor for submacular hemorrhage from choroidal neovascularization," Ophthalmology, vol. 121, no. 4, pp. 926-935, 2014.

[28] R. P. Singh, E. X. Fu, S. D. Smith, D. R. Williams, and P. K. Kaiser, "Predictive factors of visual and anatomical outcome after intravitreal bevacizumab treatment of neovascular agerelated macular degeneration: an optical coherence tomography study," The British Journal of Ophthalmology, vol. 93, no. 10, pp. 1353-1358, 2009.

[29] S. M. Hariprasad, L. S. Morse, H. Shapiro, P. Wong, and L. Tuomi, "Fixed monthly versus less frequent ranibizumab dosing and predictors of visual response in exudative agerelated macular degeneration," Journal of Ophthalmology, vol. 2012, Article ID 690641, 8 pages, 2012.

[30] J. Y. Lee, F. A. Folgar, M. G. Maguire et al., "Outer retinal tubulation in the comparison of age-related macular degeneration treatments trials (CATT)," Ophthalmology, vol. 121, no. 12, pp. 2423-2431, 2014.

[31] M. M. Altaweel, E. Daniel, D. F. Martin et al., "Outcomes of eyes with lesions composed of $>50 \%$ blood in the comparison of age-related macular degeneration treatments trials (CATT)," Ophthalmology, vol. 122, no. 2, pp. 391-398.e5, 2015.

[32] U. Mayr-Sponer, S. M. Waldstein, M. Kundi et al., "Influence of the vitreomacular interface on outcomes of ranibizumab therapy in neovascular age-related macular degeneration," Ophthalmology, vol. 120, no. 12, pp. 2620-2629, 2013.

[33] U. Schmidt-Erfurth, S. M. Waldstein, G. G. Deak, M. Kundi, and C. Simader, "Pigment epithelial detachment followed by retinal cystoid degeneration leads to vision loss in treatment of neovascular age-related macular degeneration," Ophthalmology, vol. 122, no. 4, pp. 822-832, 2015.

[34] C. Simader, M. Ritter, M. Bolz et al., "Morphologic parameters relevant for visual outcome during anti-angiogenic therapy of neovascular age-related macular degeneration," Ophthalmology, vol. 121, no. 6, pp. 1237-1245, 2014.

[35] S. M. Waldstein, M. Ritter, C. Simader, U. Mayr-Sponer, M. Kundi, and U. Schmidt-Erfurth, "Impact of vitreomacular adhesion on ranibizumab mono- and combination therapy for neovascular age-related macular degeneration," American Journal of Ophthalmology, vol. 158, no. 2, pp. 328-336.e1, 2014.

[36] G.-S. Ying, B. J. Kim, M. G. Maguire et al., "Sustained visual acuity loss in the comparison of age-related macular degeneration treatments trials," JAMA Ophthalmology, vol. 132, no. 8, pp. 915-921, 2014.

[37] R. Hoerster, P. S. Muether, V. Sitnilska, B. Kirchhof, and S. Fauser, "Fibrovascular pigment epithelial detachment is a risk factor for long-term visual decay in neovascular age-related macular degeneretion," Retina, vol. 34, no. 9, pp. 1767-1773, 2014.

[38] B. Weingessel, K. Mihaltz, and P. V. Vecsei-Marlovits, Predictors of 1-Year Visual Outcome in OCT Analysis Comparing Ranibizumab Monotherapy Versus Combination Therapy with PDT in Exsudative Age-Related Macular Degeneration, Wiener Klinische Wochenschrift, 2015.

[39] S. S. Wickremasinghe, L. Busija, R. H. Guymer, T. Y. Wong, and S. Qureshi, "Retinal venular caliber predicts visual outcome after intravitreal ranibizumab injection treatments for neovascular AMD," Investigative Ophthalmology \& Visual Science, vol. 53, no. 1, pp. 37-41, 2012.

[40] Z. Alkin, A. Ozkaya, O. A. Osmanbasoglu et al., "The role of epiretinal membrane on treatment of neovascular agerelated macular degeneration with intravitreal bevacizumab," The Scientific World Journal, vol. 2013, Article ID 958724, 7 pages, 2013.

[41] S. B. Bloch, M. la Cour, B. Sander et al., "Predictors of 1year visual outcome in neovascular age-related macular degeneration following intravitreal ranibizumab treatment," Acta Ophthalmologica, vol. 91, no. 1, pp. 42-47, 2013.

[42] J. Chhablani, I. R. Kozak, F. Mojana et al., "Fundus autofluorescence not predictive of treatment response to intravitreal bevacizumab in exudative age-related macular degeneration," Retina, vol. 32, no. 8, pp. 1465-1470, 2012.

[43] J. Chhablani, J. S. Kim, W. R. Freeman, I. Kozak, H.-Y. Wang, and L. Cheng, "Predictors of visual outcome in eyes with choroidal neovascularization secondary to age related macular degeneration treated with intravitreal bevacizumab monotherapy," International Journal of Ophthalmology, vol. 6, no. 1, pp. 62-66, 2013.

[44] C. R. Clemens, N. Bastian, F. Alten, C. Milojcic, P. Heiduschka, and N. Eter, "Prediction of retinal pigment epithelial tear in serous vascularized pigment epithelium detachment," Acta Ophthalmologica, vol. 92, no. 1, pp. e50-e56, 2014.

[45] R. M. Coco, M. R. Sanabria, M. Castrejon et al., "Funduscopic results after 4-year follow-up treatment with ranibizumab for 
age-related macular degeneration in a region of Spain," $B M C$ Ophthalmology, vol. 14, article 138, 2014.

[46] K. C. S. Fong, N. Kirkpatrick, Q. Mohamed, and R. L. Johnston, "Intravitreal bevacizumab (Avastin) for neovascular age-related macular degeneration using a variable frequency regimen in eyes with no previous treatment," Clinical \& Experimental Ophthalmology, vol. 36, no. 8, pp. 748-755, 2008.

[47] C. Framme, S. Wolf, and U. Wolf-Schnurrbusch, "Small dense particles in the retina observable by spectral-domain optical coherence tomography in age-related macular degeneration," Investigative Ophthalmology \& Visual Science, vol. 51, no. 11, pp. 5965-5969, 2010.

[48] H. M. Kang, H. J. Kwon, J. H. Yi, C. S. Lee, and S. C. Lee, "Subfoveal choroidal thickness as a potential predictor of visual outcome and treatment response after intravitreal ranibizumab injections for typical exudative age-related macular degeneration," American Journal of Ophthalmology, vol. 157, no. 5, pp. 1013.el-1021.el, 2014.

[49] S. Kang and Y.-J. Roh, "One-year results of intravitreal ranibizumab for neovascular age-related macular degeneration and clinical responses of various subgroups," Japanese Journal of Ophthalmology, vol. 53, no. 4, pp. 389-395, 2009.

[50] S. Kolb, M. Menghini, D. Barthelmes, F. Sutter, and M. KurzLevin, "The predictive value of OCT characteristics for the visual outcome in neovascular AMD," Klinische Monatsblätter für Augenheilkunde, vol. 229, no. 4, pp. 343-347, 2012.

[51] Y. H. Kwon, D. K. Lee, H. E. Kim, and O. W. Kwon, "Predictive findings of visual outcome in spectral domain optical coherence tomography after ranibizumab treatment in age-related macular degeneration," Korean Journal of Ophthalmology, vol. 28, no. 5, pp. 386-392, 2014.

[52] M. Leitritz, F. Gelisken, W. Inhoffen, M. Voelker, and F. Ziemssen, "Can the risk of retinal pigment epithelium tears after bevacizumab treatment be predicted? An optical coherence tomography study," Eye, vol. 22, no. 12, pp. 1504-1507, 2008.

[53] R. Mathew, M. Richardson, and S. Sivaprasad, "Predictive value of spectral-domain optical coherence tomography features in assessment of visual prognosis in eyes with neovascular agerelated macular degeneration treated with ranibizumab," American Journal of Ophthalmology, vol. 155, no. 4, pp. 720.e1-726.el, 2013.

[54] M. Menghini, M. M. Kurz-Levin, C. Amstutz et al., "Response to ranibizumab therapy in neovascular AMD-an evaluation of good and bad responders," Klinische Monatsblätter für Augenheilkunde, vol. 227, no. 4, pp. 244-248, 2010.

[55] Y. Nomura, H. Takahashi, X. Tan, S. Fujimura, R. Obata, and Y. Yanagi, "Effects of vitreomacular adhesion on ranibizumab treatment in Japanese patients with age-related macular degeneration," Japanese Journal of Ophthalmology, vol. 58, no. 5, pp. 443-447, 2014.

[56] A. Oishi, M. Shimozono, M. Mandai, M. Hata, A. Nishida, and Y. Kurimoto, "Recovery of photoreceptor outer segments after anti-VEGF therapy for age-related macular degeneration," Graefe's Archive for Clinical and Experimental Ophthalmology, vol. 251, no. 2, pp. 435-440, 2013.

[57] O. Shona, B. Gupta, R. Vemala, and S. Sivaprasad, "Visual acuity outcomes in ranibizumab-treated neovascular age-related macular degeneration; stratified by baseline vision," Clinical and Experimental Ophthalmology, vol. 39, no. 1, pp. 5-8, 2011.
[58] M. Suzuki, N. Nagai, K. Izumi-Nagai et al., "Predictive factors for non-response to intravitreal ranibizumab treatment in agerelated macular degeneration," The British Journal of Ophthalmology, vol. 98, no. 9, pp. 1186-1191, 2014.

[59] L. A. Toth, M. Stevenson, and U. Chakravarthy, "ANTI-vascular endothelial growth factor therapy for neovascular age-related macular degeneration," Retina, vol. 35, no. 10, pp. 1957-1963, 2015.

[60] T. H. C. Tran, G. Querques, G. Forzy, and E. H. Souied, "Angiographic regression patterns after intravitreal ranibizumab injections for neovascular age-related macular degeneration," Ophthalmic Surgery Lasers and Imaging, vol. 42, no. 6, pp. 498-508, 2011.

[61] G. Ö. Üney, N. Ünlü, M. A. Acar et al., "Role of posterior vitreous detachment on outcome of anti-vascular endothelial growth factor treatment in age-related macular degeneration," Retina, vol. 34, no. 1, pp. 32-37, 2014.

[62] J. Z. Nowak, "Age-related macular degeneration (AMD): pathogenesis and therapy," Pharmacological Reports, vol. 58, no. 3, pp. 353-363, 2006.

[63] B. G. Busbee, A. C. Ho, D. M. Brown et al., "Twelve-month efficacy and safety of $0.5 \mathrm{mg}$ or $2.0 \mathrm{mg}$ ranibizumab in patients with subfoveal neovascular age-related macular degeneration," Ophthalmology, vol. 120, no. 5, pp. 1046-1056, 2013.

[64] C. D. Regillo, D. M. Brown, P. Abraham et al., "Randomized, double-masked, sham-controlled trial of ranibizumab for neovascular age-related macular degeneration: PIER Study year 1," American Journal of Ophthalmology, vol. 145, no. 2, pp. 239248.e5, 2008.

[65] P. Abraham, H. Yue, and L. Wilson, "Randomized, doublemasked, sham-controlled trial of ranibizumab for neovascular age-related macular degeneration: PIER study year 2," The American Journal of Ophthalmology, vol. 150, no. 3, pp. 315.el324.e1, 2010.

[66] D. F. Martin, M. G. Maguire, S. L. Fine et al., "Ranibizumab and bevacizumab for treatment of neovascular age-related macular degeneration: two-year results," Ophthalmology, vol. 119, no. 7, pp. 1388-1398, 2012.

[67] P. U. Dugel, "Anti-PDGF combinationtherapy in noevascular age-related macular degeneration: results of a phase $2 \mathrm{~b}$ study," Retina Today, pp. 65-71, 2013.

[68] P. Carmeliet, "Mechanisms of angiogenesis and arteriogenesis," Nature Medicine, vol. 6, no. 4, pp. 389-395, 2000.

[69] L. E. Benjamin, I. Hemo, and E. Keshet, "A plasticity window for blood vessel remodelling is defined by pericyte coverage of the preformed endothelial network and is regulated by PDGF-B and VEGF," Development, vol. 125, no. 9, pp. 1591-1598, 1998.

[70] S. B. Bloch, H. Lund-Andersen, B. Sander, and M. Larsen, "Subfoveal fibrosis in eyes with neovascular age-related macular degeneration treated with intravitreal ranibizumab," American Journal of Ophthalmology, vol. 156, no. 1, pp. 116.el-124.e1, 2013.

[71] J. C. Bonner, "Regulation of PDGF and its receptors in fibrotic diseases," Cytokine and Growth Factor Reviews, vol. 15, no. 4, pp. 255-273, 2004.

[72] R. S. Kaiser, "Anti-PDGF combination therapy for AMD," Retina Today, pp. 68-70, 2013.

[73] J. S. Heier, D. M. Brown, V. Chong et al., "Intravitreal aflibercept (VEGF trap-eye) in wet age-related macular degeneration," Ophthalmology, vol. 119, no. 12, pp. 2537-2548, 2012.

[74] H. Cho, C. P. Shah, M. Weber, and J. Heier, "Aflibercept for exudative AMD with persistent fluid on ranibizumab and/or 
bevacizumab," The British Journal of Ophthalmology, vol. 97, no. 8, pp. 1032-1035, 2013.

[75] D. Pauleikhoff and B. Kirchhof, "Retreatment criteria in antiVEGF therapy of exudative AMD: critical analysis of present regimes and new morphological definition of 'lesion activity," Graefe's Archive for Clinical and Experimental Ophthalmology, vol. 249, no. 5, pp. 631-632, 2011.

[76] H. Gerding, V. Loukopoulos, J. Riese, L. Hefner, and M. Timmermann, "Results of flexible ranibizumab treatment in agerelated macular degeneration and search for parameters with impact on outcome," Graefe's Archive for Clinical and Experimental Ophthalmology, vol. 249, no. 5, pp. 653-662, 2011. 


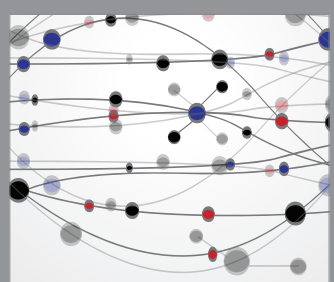

The Scientific World Journal
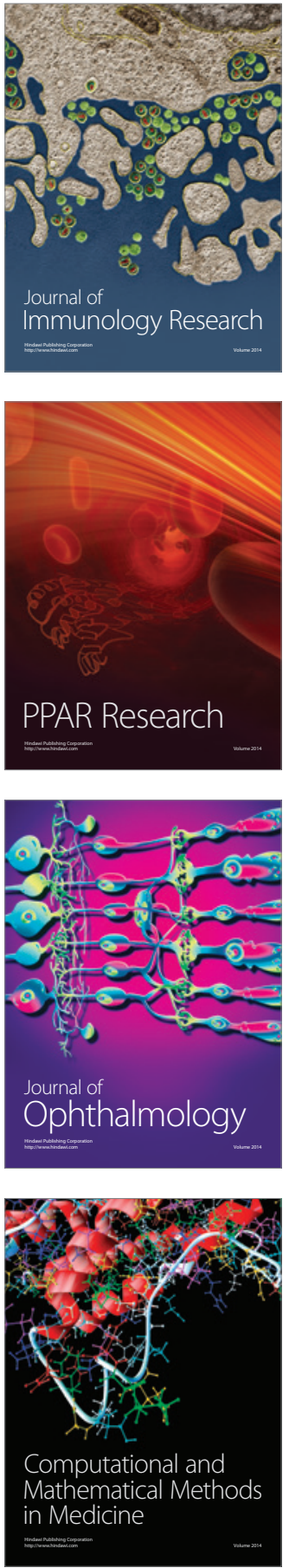

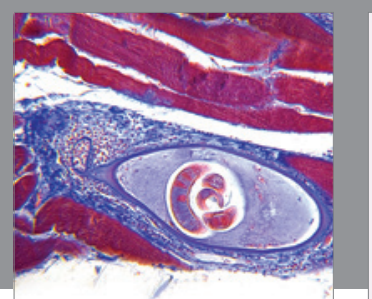

Gastroenterology Research and Practice

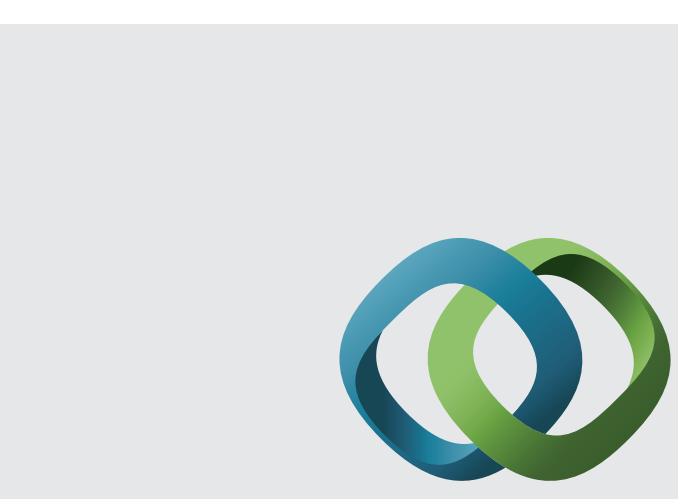

\section{Hindawi}

Submit your manuscripts at

http://www.hindawi.com
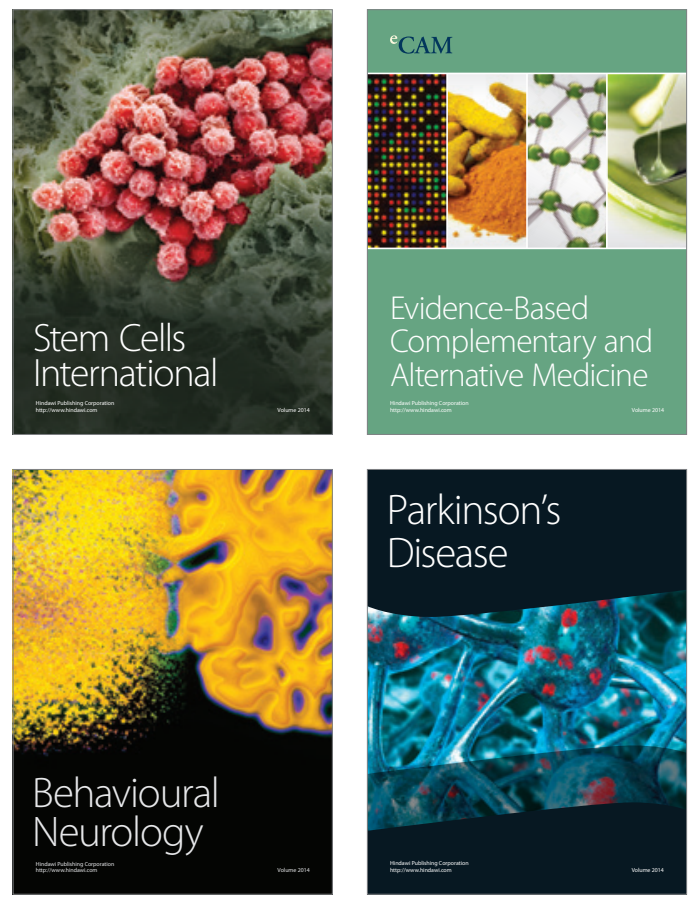
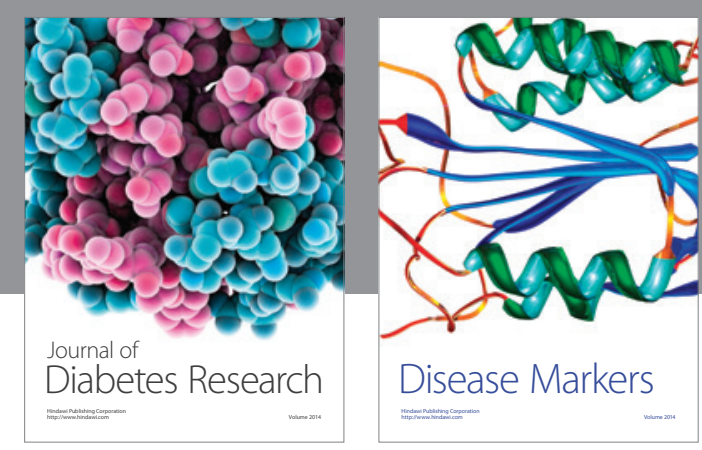

Disease Markers
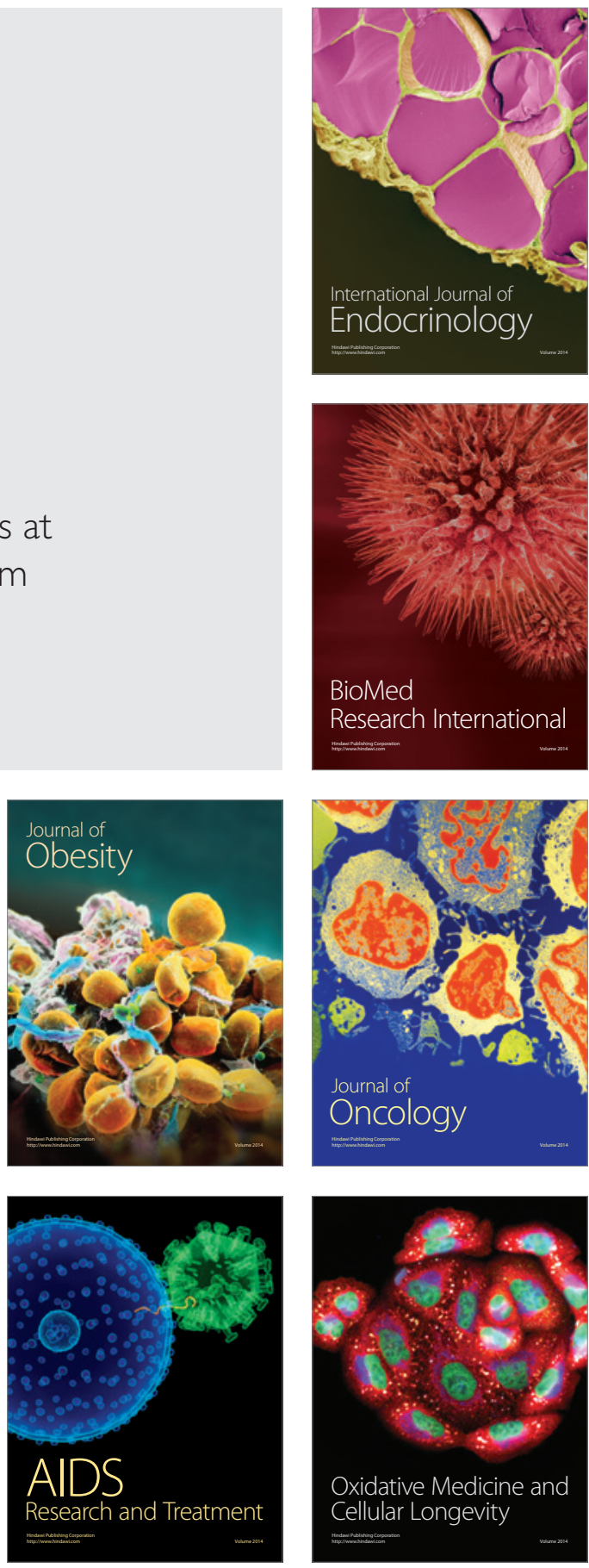